\title{
ON THE ENVELOPE OF THE AXES OF A SYSTEM OF CONICS PASSING THROUGH THREE FIXED POINTS*
}

BY

R. E. ALLARDICE

In a recent number of the Annals of Mathematics $\nmid$ I have shown that the envelope of the asymptotes of a system of conics passing through three fixed points consists of two three-cusped hypocycloids, touching the three straight lines that join the three fixed points in pairs. I propose now to show that the envelope of the axes of the same system of conics consists of two three-cusped hypocycloids touching three concurrent straight lines.

The foci of a conic may be regarded as four of the vertices of a complete four-side circumscribing the conic, the other two vertices being the circular points at infinity; then the straight line at infinity is one diagonal line of this four-side, and the axes are the other two diagonal lines.

The coördinates of the circular points at infinity are $\left(1,-e^{c i},-e^{-B i}\right)$ and $\left(1,-e^{-C i},-e^{B i}\right)$; let us denote these points for the present by $\left(x_{1}, y_{1}, z_{1}\right)$ and $\left(x_{2}, y_{2}, z_{2}\right)$.

Let the equation of the conic be

$$
U \equiv \lambda_{1} y z+\lambda_{2} z x+\lambda_{3} x y=0
$$

the three fixed points through which the conic is to pass being the vertices of the triangle of reference; and put

$$
\begin{aligned}
& U_{1} \equiv \lambda_{1} y_{1} z_{1}+\lambda_{2} z_{1} x_{1}+\lambda_{3} x_{1} y_{1} ; \quad U_{2}=\lambda_{1} y_{2} z_{2}+\lambda_{2} z_{2} x_{2}+\lambda_{3} x_{2} y_{2} \\
& U_{1}^{\prime} \equiv x_{1} \frac{\partial U}{\partial x}+y_{1} \frac{\partial U}{\partial y}+z_{1} \frac{\partial U}{\partial z} ; \quad U_{2}^{\prime}=x_{2} \frac{\partial U}{\partial x}+y_{2} \frac{\partial U}{\partial y}+z_{2} \frac{\partial U}{\partial z}
\end{aligned}
$$

\footnotetext{
* Presented to the Society (Chicago) January 2, 1903. Received for pablication August 2, 1902.

† On some curves connected with a system of similar conics, Annals of Mathematics, 2d series, vol. 3 (1902), p. 154.
} 
The equations of the tangents from the circular points at infinity are

and

$$
U_{1}^{\prime 2}=4 U U_{1}
$$

$$
U_{2}^{\prime 2}=4 U U_{2}
$$

and the foci are the intersections of these two pairs of straight lines.

The equation $U_{2}{U_{1}^{\prime}}^{2}=U_{1}{U_{2}^{\prime 2}}^{2}$ evidently represents a third pair of straight lines passing through the foci, and must therefore represent the axes.

Now the condition for similarity may be expressed in the form *

$$
\sum\left(\lambda_{1}^{2} \sin ^{2} A-2 \lambda_{2} \lambda_{3} \sin B \sin C\right)=t^{2}\left(\lambda_{1} \cos A+\lambda_{2} \cos B+\lambda_{3} \cos C\right)^{2},
$$

where $t$ is the tangent of the angle between the asymptotes; or,

that is,

$$
\sum\left(\lambda_{1}^{2}-2 \lambda_{2} \lambda_{3} \cos A\right)=s^{2}\left(\lambda_{1} \cos A+\lambda_{2} \cos B+\lambda_{3} \cos C\right)^{2},
$$

$$
U_{1} U_{2}=s^{2} P^{2},
$$

where $s$ is the secant of the angle between the asymptotes, and

$$
P=\lambda_{1} \cos A+\lambda_{2} \cos B+\lambda_{3} \cos C .
$$

Hence the equations of the axes may be expressed in the form

and

$$
U_{1} U_{2}^{\prime}=s P U_{1}^{\prime}, \quad \text { or } \quad U_{2} U_{1}^{\prime}=s P U_{2}^{\prime} ;
$$

$$
U_{1} U_{2}^{\prime}=-s P U_{1}^{\prime}, \quad \text { or } \quad U_{2} U_{1}^{\prime}=-s P U_{2}^{\prime} \text {. }
$$

Using the first of these equations, we may write the tangential coördinates of the corresponding axis in the form

Noticing that

$$
\begin{aligned}
u & =U_{1}\left(\lambda_{2} z_{2}+\lambda_{3} y_{2}\right)-s P\left(\lambda_{2} z_{1}+\lambda_{3} y_{1}\right), \\
v & =U_{1}\left(\lambda_{3} x_{2}+\lambda_{1} z_{2}\right)-s P\left(\lambda_{3} x_{1}+\lambda_{1} z_{1}\right), \\
w & =U_{1}\left(\lambda_{1} y_{2}+\lambda_{2} x_{2}\right)-s P\left(\lambda_{1} y_{1}+\lambda_{2} x_{1}\right) .
\end{aligned}
$$

$$
\lambda_{1}\left(y_{1} z_{2}+y_{2} z_{1}\right)+\lambda_{2}\left(z_{1} x_{2}+z_{2} x_{1}\right)+\lambda_{3}\left(x_{1} y_{2}+x_{2} y_{1}\right)=-2 P,
$$

we have, on multiplying these equations first by $x_{1}, y_{1}, z_{1}$ and adding, and then by $x_{2}, y_{2}, z_{2}$, and adding,

$$
\begin{aligned}
& V \equiv x_{1} u+y_{1} v+z_{1} w=-2 P U_{1}-2 s P U_{1}, \\
& W=x_{2} u+y_{2} v+z_{2} w=2 U_{1} U_{2}+2 s P^{2} .
\end{aligned}
$$

* See the paper entitled, On some curves, etc., referred to above. 
Hence, taking account of the relation $U_{1} U_{2}=s^{2} P^{2}$, we find,

$$
P=\frac{V}{\sqrt{2 s(s+1)}}, \quad U_{1}=-\frac{V \vee s}{\sqrt{2(s+1) W}} .
$$

Now writing the coördinates in the form

$$
\begin{aligned}
& u=\left(z_{2} U_{1}-z_{1} s P\right) \lambda_{2}+\left(y_{2} U_{1}-y_{1} s P\right) \lambda_{3}, \\
& v=\left(z_{2} U_{1}-z_{1} s P\right) \lambda_{1}+\left(x_{2} U_{1}-x_{1} s P\right) \lambda_{3}, \\
& w=\left(y_{2} U_{1}-y_{1} s P\right) \lambda_{1}+\left(x_{2} U_{1}-x_{1} s P\right) \lambda_{2}
\end{aligned}
$$

and using the equation

$$
P=\lambda_{1} \cos A+\lambda_{2} \cos B+\lambda_{3} \cos C,
$$

we have, on eliminating $\lambda_{1}, \lambda_{2}, \lambda_{3}$ the relation

$$
\left|\begin{array}{cccc}
u & 0 & z_{2} U_{1}-z_{1} s P & y_{2} U_{1}-y_{1} s P \\
v & z_{2} U_{1}-z_{1} s P & 0 & x_{2} U_{1}-x_{1} s P \\
w & y_{2} U_{1}-y_{1} s P & x_{2} U_{1}-x_{1} s P & 0 \\
P & \cos A & \cos B & \cos C
\end{array}\right|=0
$$

On substituting the values of $P$ and $U_{1}$ found above, and reducing by means of the relations

$$
x_{2} V+x_{1} W=2(u-v \cos C-w \cos B), \text { etc. }
$$

we finally obtain the equation of the envelope in the form

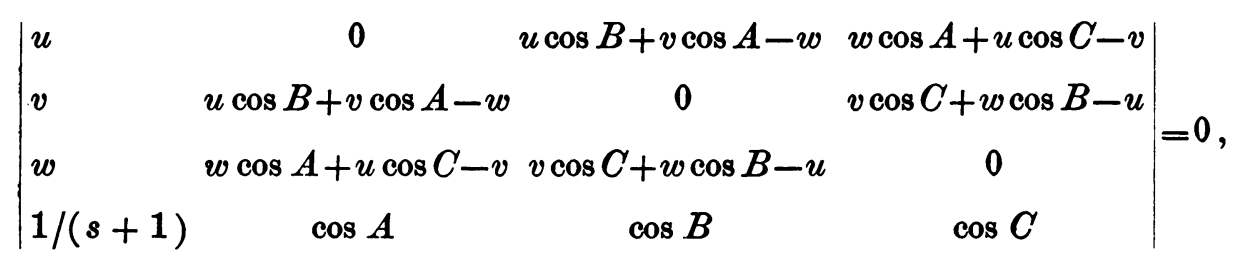

or

$\sum[u(v \cos C+w \cos B-u)\{u \cos (B-C)-v \cos B-w \cos C\}]$

$-\frac{2}{s+1}(v \cos C+w \cos B-u)(w \cos A+u \cos C-v)(u \cos B+v \cos A-w)=0$.

It may be shown that this curve has the straight line at infinity for a double tangent, the circular points at infinity being the points of contact. 
It must therefore be of the fourth order and have three cusps; and hence for all values of $s$ (except $s=-1$ ) it is a three-cusped hypocycloid.

It may easily be shown that it always touches the perpendicular bisectors of the sides of the triangle of reference; in the special case, $s=-1$, the curve degenerates into the points at infinity on these three lines.

The two axes envelope the same curve only in the case of the equilateral hyperbola, for which $s=\infty$.

Stanford University, California. 